\title{
Porphyrin-Based Tumor-Targeting Theranostic Agent: Gd-TDAP
}

\author{
Soyeon $\mathrm{Kim}^{+,}$, Ji-ung Yang ${ }^{+, \neq}$, Jae Hun Ahn ${ }^{+, \Phi}$, In Ok $\mathrm{Ko}^{+}$, Jung Young $\mathrm{Kim}^{+}$, Yong Jin Lee ${ }^{\dagger}$, Ji-Ae Park ${ }^{+, *}$ \\ ${ }^{\dagger}$ Division of Applied RI, Korea Institute of Radiological \& Medical Sciences (KIRAMS), Seoul 01812, Korea \\ ${ }^{\ddagger}$ Department of Medical \& Biological Engineering, Kyungpook National University, Daegu 41566, Korea \\ ${ }^{9}$ Graduate School of Translational Medicine, Seoul National University College of Medicine, Seoul 03080, Korea
}

\section{Materials and methods}

General Remarks.

All chemical reagents were purchased from Sigma-Aldrich (St, Louis, MO, USA) and used without further purifications, the other cases are indicated separately. Deionized water was used in all cases. High resolution FAB mass (HR-FAB-MS) was measured by using a JMS-700 model (Jeol, Japan) mass spectrophotometer by Korea Basic Science Institute (KBSI). The purity of Gd-TDAP was determined to be above $90 \%$ by HPLC (high performance liquid chromatography, Waters) system equipped with an Atlantis C18 column $(150 \mathrm{X} 3.0 \mathrm{~mm})$ was used for the purity test. The method for purity test were as follows: eluent A, $0.1 \%$ TFA in ACN; B, $0.1 \%$ TFA in water; gradient, $50 \%$ to $100 \% \mathrm{~A}$ in $30 \mathrm{~min}$; flow rate $0.5 \mathrm{~mL} / \mathrm{min}$ ) Fluorescence imaging was acquired by a Maestro system (CRI, Inc., Blue filter: Excitation filter, 445 to $490 \mathrm{~nm}$; Emission filter, $515 \mathrm{~nm}$ longpass) and an IVIS Imaging System (IVIS Spectrum, Perkin Elmer, excitation filter, $430 \mathrm{~nm}$; emission filter, $680 \mathrm{~nm}$ ).

\section{Characterization of Gd-TDAP}

Hydroxo-gadolinium(III) 5,10,15,20-(tetra-N,N-dimethyl-4-aminophenyl)porphyrin (Gd-TDAP) was purchased from PorphyChem (Lot Number; 158Abo18, France). HR-FAB-MS (m/z): calced for $\mathrm{C}_{52} \mathrm{H}_{48} \mathrm{~N}_{8} \mathrm{Gd}, 942.3243\left([\mathrm{M}-\mathrm{OH}]^{+}\right)$; found, $942.3249\left([\mathrm{M}-\mathrm{OH}]^{+}\right)$.

\section{Relaxivity.}

The samples were prepared by diluting $1 \mathrm{mM}$ DMSO/water (10\% DMSO) stock solution with D.I. water to final concentrations: $1,0.5,0.25,0125$ and $0.0625 \mathrm{mM}$. Relaxation was measured with a $3.0 \mathrm{~T}$ MRI system (Magnetom Tim Trio, Siemens, Germany). $T_{1}$ measurements were performed using an inversion recovery sequence with 35 different inversion times (TIs) between 50 and $1750 \mathrm{msec}$. $T_{2}$ measurements were acquired using a CPMG (Carr-Purcell-MeiboonGill) pulse sequence with 32 different echo times (TEs) ranging from 33.8 to $1081.6 \mathrm{msec} . T_{1}$ and $T_{2}$ relaxation times were calculated from a non-linear least square fit of the signal intensity measured at each TI or TE value. The relaxation rates $\left(R_{1}\right.$ and $\left.R_{2}\right)$ are the reciprocal of the relaxation times $\left(T_{1}\right.$ and $\left.T_{2}\right)$, and the relaxation rates according to the concentrations was linearly fitted, and the relaxivities $\left(r_{1}\right.$ and $\left.r_{2}\right)$ were obtained from the slope.

\section{Determination of binding constant with HSA.}

This experiment was carried out with minor modifications according to the published literature method. ${ }^{3}$ Thirteen different concentrations $(0 \sim 3 \mathrm{mM})$ of samples were prepared by diluting the $25 \mathrm{mM}$ stock solution of Gd-TDAP (in $30 \%$ DMSO, 15\% Tween 80 and 55\% saline) in $0.67 \mathrm{mM}$ HSA solution. The proton paramagnetic relaxation rates were measured in the same way as relaxivity method. The change of relaxation rate according to the $\mathrm{Gd}$ concentration was fitted using equation 1 to obtain binding constant $\left(\mathrm{K}_{\mathrm{a}}\right)$ with HSA. $p^{0}=$ the concentration of the HSA; $s^{0}=$ the paramagnetic complex concentration; $N=$ the number of independent interaction sites ( $N$ was set to 1$) ; r_{1}{ }^{\mathrm{c}}=$ the relaxivities of the complex HSA-contrast agent; $r_{1}{ }^{\mathrm{f}}=$ the relaxivities of the free contrast agent. 


$$
R_{1}^{p^{o b s}}=1000 \times\left[\left(r_{1}^{f} s^{0}\right)+\frac{1}{2}\left(r_{1}^{c}-r_{1}^{f}\right)\left\{\left(N_{p}^{0}\right)+s^{0}+K_{a}^{-1}-\sqrt{\left(\left(N_{p}^{0}\right)+s^{0}+K_{a}^{-1}\right)^{2}-4 N s^{0} p^{0}}\right\}\right]
$$

\section{Octanol-Water partition Coefficient.}

Two samples ( $1 \mathrm{mg}$ of Gd-TDAP in each vial) were mixed with $1 \mathrm{ml}$ of 1-octanol and $1 \mathrm{ml}$ of water, then gently mixed for 2 days using a vortex mixer. Then the vials were placed on a flat surface so that the mixture was divided into two layers for 24 hours. The divided layers were separated, the Gd (III) amount of each layer was measured through inductively coupled plasma mass spectrometry (ICP-MS, Nexion 350D, Perkin Elmer, USA), and the octanol-water partition coefficient was calculated as follows; $\log P_{\text {oct } / \text { wat }}=\log \left(C_{\mathrm{o}} / C_{\mathrm{w}}\right)$, where $C_{\mathrm{o}}$ and $C_{\mathrm{w}}$ are the concentration of Gd (III) species in the octanolrich and water-rich layer, respectively.

\section{In vitro fluorescence measurement}

The absorbance and emission spectrum of GdL were measured by SpectraMax i3x (Molecular Devices, CA, U.S.). Serum stability assay was performed with slight modification of literature method. ${ }^{4}$ Stock solutions were made with $0.2 \mathrm{mg}$ of $\mathrm{L}$ or GdL dissolved in DMSO (10\%), tween 80 (15\%) and water (75\%). $50 \mu \mathrm{L}$ of each stock solution was added to water $(950 \mu \mathrm{L})$ and HSA solution $(0.67 \mathrm{mM}, 950 \mu \mathrm{L})$. The emission spectrum was measured immediately after the stock solution was added and every $20 \mathrm{~min}$ for 1 hour.

\section{Cell culture}

Human malignant glioma cell (U87MG, ATCC ${ }^{*}$ HTB-14) was cultured in growth medium, containing Minimum Essential Medium alpha (Corning, NY) medium supplemented with 10\% (v/v) fetal bovine serum (FBS, JR Scientific Inc., USA) and $1 \%$ antibiotics-antimycotics (Gibco, USA). Cells were incubated at 37 under $5 \% \mathrm{CO}_{2} / 95 \%$ air atmosphere.

\section{Cell Fractions}

This experiment was carried out with reference to published papers. ${ }^{4} \mathrm{U} 87 \mathrm{MG}$ cells were plated $3 \times 10^{6}$ in $100 \mathrm{~mm}$ dishes in MEM-alpha growth medium. The medium was removed, and then Gd-TDAP (100 $\mu \mathrm{M})$ in MEM-alpha serum depletion media was incubated for $24 \mathrm{~h}$. The cells were harvested and centrifuged at $500 \times \mathrm{g}$ for $5 \mathrm{~min}$ then washed with PBS. For cell fractionation, NE-PER Nuclear and Cytoplasmic Extraction Reagents Kit (Thermo, VA, USA) was used according to the manufacturer's instructions. Cells not treated with the drug were also fractionated and used as a control. First, the cell pellet was re-suspended in $200 \mu \mathrm{L}$ of ice-cold CER-I reagent and then completely suspended by vortexing for 15 sec at the highest setting. After incubation for $10 \mathrm{~min}$ on ice, ice-cold CER-II reagent $(11 \mu \mathrm{L})$ was added and then vortexed the tube for $5 \mathrm{sec}$ at the highest setting. After incubating for $1 \mathrm{~min}$ on ice and vortexing for $5 \mathrm{sec}$, the tube was centrifuged for $5 \mathrm{~min}$ in a microcentrifuge $(16,000 \times \mathrm{g})$. The supernatant, cytoplasmic extraction, was immediately transferred to a pre-chilled tube. The insoluble pellet was re-suspended in ice-cold NER reagent $(100 \mu \mathrm{L})$ then the tube was vortexed for 5 sec and centrifuged for $5 \mathrm{~min}(16,000 \times \mathrm{g})$. Vortex the tube for $15 \mathrm{sec}$ and then put on ice for $10 \mathrm{~min}$ was repeated for a total of 40 $\min$. The tube was then centrifuged at $16,000 \times \mathrm{g}$ for $10 \mathrm{~min}$, and the supernatant, nuclear extract was transferred to a prechilled tube. The cytoplasmic, nuclear extract and the last pellet which contains membrane parts were stored at $-80 \bigotimes$ until use.

\section{In vitro MR imaging}

After cell fraction, each sample was diluted with $500 \mu \mathrm{L}$ of PBS to obtain MR $T_{1}$-weighted images. Images were taken using a wrist coil by $3 \mathrm{~T}$ MR unit. The imaging parameters for turbo spin echo (TSE) $T_{1}$-weighted images were as follows: $\mathrm{TR}=$ 500 ms; TE = 12 ms; 60 mm FOV; 256 x 204 matrix size; ETL = 5; slice thickness = 3 mm; NEX = 10.

\section{Brain Tumor model}

All animal experiments involving the use of mice were performed according to a protocol approved by Korea Institute of Radiological \& Medical Sciences (KIRAMS). This experiment was performed according to a published literature with 
slight modification..$^{5-6}$

For cell injection, U87 MG cells were harvested during the log phase of growth and resuspended in serum-free MEM alpha $\left(1 \times 10^{6}\right.$ cells $\left./ 5 \mu \mathrm{L}\right)$. Nude mice (Foxn/nu, 6-week-old, male) were anesthetized by $3 \%$ isoflurane and placed in a stereotactic frame. After a midline incision, a burr hole was made by drilled in the skull $(1 \mathrm{~mm}$ anterior and $2 \mathrm{~mm}$ right from the bregma). The prepared cell suspension was injected in the mice brain striata using Hamilton syringe (depth: 3 $\mathrm{mm}$, injection rate: $1 \mu \mathrm{L} / \mathrm{min}$ ). After waiting for $3 \mathrm{~min}$ post injection, the syringe needle was slowly removed, then the burr hole was sealed with dental cement and the incision was sutured and sterilized with iodine pads. The mice were allowed to recover in warmed-up cages and then placed in the vivarium.

\section{ex vivo Fluorescence imaging}

The brain tumor model mice $(18 \sim 25 \mathrm{~g})$ were anesthetized and sacrificed at $15 \mathrm{~min}$ after intravenous injection of GdTDAP $(0.05 \mathrm{mmol} / \mathrm{kg})$. After perfusion to remove whole blood, brains from each mouse were collected and tumor and normal regions were separated into similar sizes. The tissues were monitored using a IVIS Imaging System (IVIS Spectrum, Perkin Elmer, excitation filter, $430 \mathrm{~nm}$; emission filter, $680 \mathrm{~nm}$ ). After ex vivo fluorescence imaging, the amount of Gd present in each tissue was measured via ICP/MS (Nexion 350D, Perkin Elmer, USA).

\section{MR Imaging and Image Analysis}

Brain tumor region was confirmed by $T_{2}$-weighted MR image 2-3 weeks after the injection of cancer cells, and then an in vivo MR imaging was conducted. During MR imaging, mice were anesthetized with $1.5 \%$ isoflurane in oxygen. After each measurement, the mice were revived from anesthesia and stayed in cages containing free water and food.

Brain MR images were obtained with a 3 T MR unit (Magnetom Tim trio, Siemens, Germany) equipped with a homemade RF coil for animal brain imaging. The imaging parameters for turbo spin echo (TSE) $T_{2}$-weighted images were as follows: repetition time $(\mathrm{TR})=3000 \mathrm{~ms}$; echo time $(\mathrm{TE})=39 \mathrm{~ms} ; 50 \mathrm{~mm}$ field of view $(\mathrm{FOV}) ; 256 \mathrm{x} 180$ matrix size; echo train length $(\mathrm{ETL})=10$; slice thickness $=0.8 \mathrm{~mm}$; number of averages $(\mathrm{NEX})=3$. The imaging parameters for TSE $T_{1}$-weighted images were as follows: $\mathrm{TR}=580 \mathrm{~ms}$; TE $=13 \mathrm{~ms} ; 40 \mathrm{~mm}$ FOV; $192 \times 154$ matrix size; ETL = 3; slice thickness $=0.8 \mathrm{~mm}$; $\mathrm{NEX}=8$.

The whole body imaging parameters for TSE $T_{1}$-weighted images were as follows: $\mathrm{TR}=580 \mathrm{~ms}$; TE $=12 \mathrm{~ms} ; 65 \mathrm{~mm} \mathrm{FOV}$; $192 \times 154$ matrix size; $\mathrm{ETL}=5$; slice thickness $=1 \mathrm{~mm}$; NEX $=3$.

The signal intensities of specific regions in MR image were measured using program imageJ (National Institutes of Health, USA). The contrast to noise ratio (CNR) was calculated using eq. 2, where SNR is the signal to noise ratio.

$$
\mathrm{CNR}=\mathrm{SNR}_{\text {post }}-\mathrm{SNR}_{\text {pre }}
$$

(a)

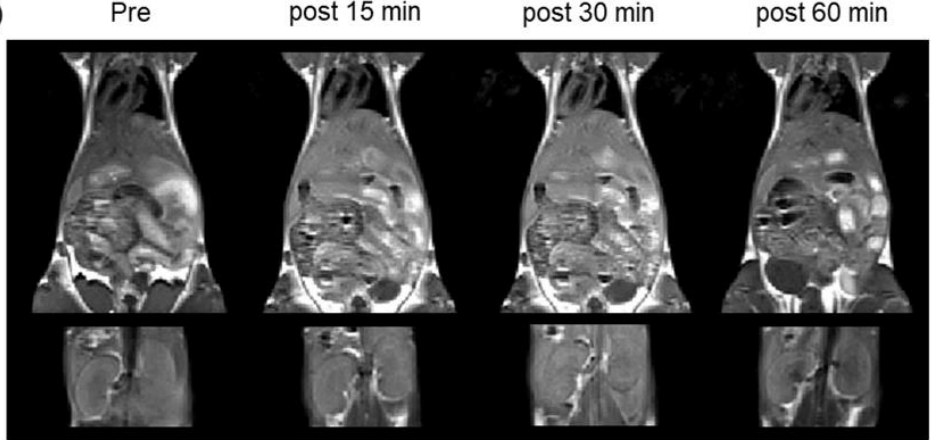

(b)

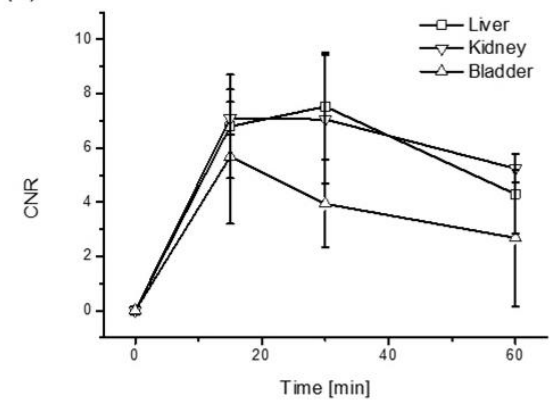

Figure S1. (a) Coronal $T_{1}$-weighted MR images and (b) CNR of liver, kidney and bladder before and after injection of Gd-TDAP (0.05 mmol/kg, i.v. injection). Data are shown as the mean $\pm \mathrm{SD}(\mathrm{n}=2)$. 


\section{References}

1. Schaverien, C. J., Octaethylporphyrin-yttrium-methyl chemistry: preparation and selective activation of dioxygen by (oep) Y ( $\mu$-Me) 2AlMe2. Journal of the Chemical Society, Chem. Commun. 1991, 7, 458-460.

2. Buckley, H. L.; Arnold, J., Recent developments in out-of-plane metallocorrole chemistry across the periodic table. Dalton Transactions 2015, 44 (1), 30-36.

3. Caravan, P.; Cloutier, N. J.; Greenfield, M. T.; McDermid, S. A.; Dunham, S. U.; Bulte, J. W.; Amedio, J. C.; Looby, R. J.; Supkowski, R. M.; Horrocks, W. D., The interaction of MS-325 with human serum albumin and its effect on proton relaxation rates. J. Am. Chem. Soc. 2002, 124 (12), 3152-3162.

4. Kim, H. K.; Kang, M. K.; Jung, K. H.; Kang, S. H.; Kim, Y. H.; Jung, J. C.; Lee, G. H.; Chang, Y.; Kim, T. J., Gadolinium complex of DO3A-benzothiazole aniline (BTA) conjugate as a theranostic agent. J Med Chem 2013, 56 (20), 8104-8111.

5. Yin, Y.; Wang, J.; Yang, M.; Du, R.; Pontrelli, G.; McGinty, S.; Wang, G.; Yin, T.; Wang, Y., Penetration of the blood-brain barrier and the anti-tumour effect of a novel PLGA-lysoGM1/DOX micelle drug delivery system. Nanoscale 2020, 12 (5), 2946-2960.

6. Jung, K. H.; Kim, H. K.; Lee, G. H.; Kang, D. S.; Park, J. A.; Kim, K. M.; Chang, Y.; Kim, T. J., Gd complexes of macrocyclic diethylenetriaminepentaacetic acid (DTPA) biphenyl-2,2'-bisamides as strong blood-pool magnetic resonance imaging contrast agents. J. Med. Chem. 2011, 54 (15), 5385-94. 